\title{
Induction of a C-repeat binding factor gene by calcium to resist low temperature stress in rice
}

\author{
Gopal Chowdhary and Chanakya Nath Kundu* \\ KIIT School of Biotechnology, KIIT University, Campus-11, Patia, Bhubaneswar, Odisha, 751024, India. \\ Accepted 27 June, 2012

\begin{abstract}
Adaptation to stress is an important phenomenon in plants. C-repeat binding factor (CBF), an Apetela 2 domain transcriptional activator, helps the plant in combating cold stress. Here, we cloned and purified a CBF family gene (OsDREB1B) from Oryza sativa. This gene (OsDREB1B) is induced by calcium in absence of cold. The bioinformatics analysis reveals that the induction of OsDREB1B is probably mediated through an EF hand calcium binding protein. This result establishes a link between rise in calcium and CBF induction in cold acclimation.
\end{abstract}

Key words: C-repeat binding factor (CBF), abiotic stress, calcium, CBF regulon.

\section{INTRODUCTION}

Plants are exposed to various environmental stresses such as drought, salinity, metal and temperature. Being sessile in nature, they cannot escape from stress conditions, rather they develop mechanism to combat the stress. Hence, in order to survive plants develop various degree of tolerance to these environmental stresses. Low temperature (LT) conditions being prevalent across the globe permanently or temporarily is an important area of study. Degree of tolerance to freeze and low temperature varies from plant to plant (Thomashow, 2010).

The transcriptional activator C-repeat binding factor (CBF) is known to play an important role in cold tolerance in plants. $\mathrm{CBF}$ is an Apetela 2 domain containing transcriptional factor and is induced upon cold stress (Gilmour et al., 1998). CBF regulates a battery of genes referred as "CBF regulon". CBF2 over expression in Arabidopsis thaliana leads to up-regulation of 302 genes and down-regulation of 212 genes (Vogel et al., 2005). One of the main targets of CBF is cold regulated gene family which stabilizes the membrane and helps in combating cold stress (Zhao et al., 2008). The induction of CBF is under the control of another constitutively expressed transcriptional activator called Inducer of CBF

*Corresponding author. E-mail: cnkundu@gmail.com. Tel: +910674-272-5466. Fax: +91-0674-272-5732. expression (ICE). ICE is activated by interplay of LT dependent phosphorylation/dephosphorylation cascade (Chinnusamy et al., 2003). Monroy and Dhindsa (1995) have shown that LT increases cytosolic calcium, inhibits a protein phosphatase $2 \mathrm{~B}$ and up-regulates Cold Acclimation Specific (CAS) gene. This suggests that most upstream event in LT signaling occur through elevated levels of cytosolic calcium, but a direct link between induction of CBF gene and elevated cytosolic calcium is missing. In this present communication an effort has been made to delineate the link between elevated levels of cytosolic calcium and CBF induction using rice as a model system.

\section{MATERIALS AND METHODS}

\section{Cloning of LT inducible CBF from Oryza}

A CBF gene from Oryza sativa Indica was cloned using Reverse Transcriptase Polymerase Chain (RT-PCR) reaction. Seven day old seedlings of rice were exposed to $\mathrm{LT}\left(4^{\circ} \mathrm{C}\right)$ for $2 \mathrm{~h}$ followed by RNA extraction and amplification by RT-PCR. The $0.6 \mathrm{~kb}$ long amplified CDNA fragment was cloned in pGEM-T Easy vector (Promega, USA) and sequenced. It was found to be similar to OsDREB1B (NCBI Accession no. AF300972; Dubouzet et al., 2003). For the isolation and purification 


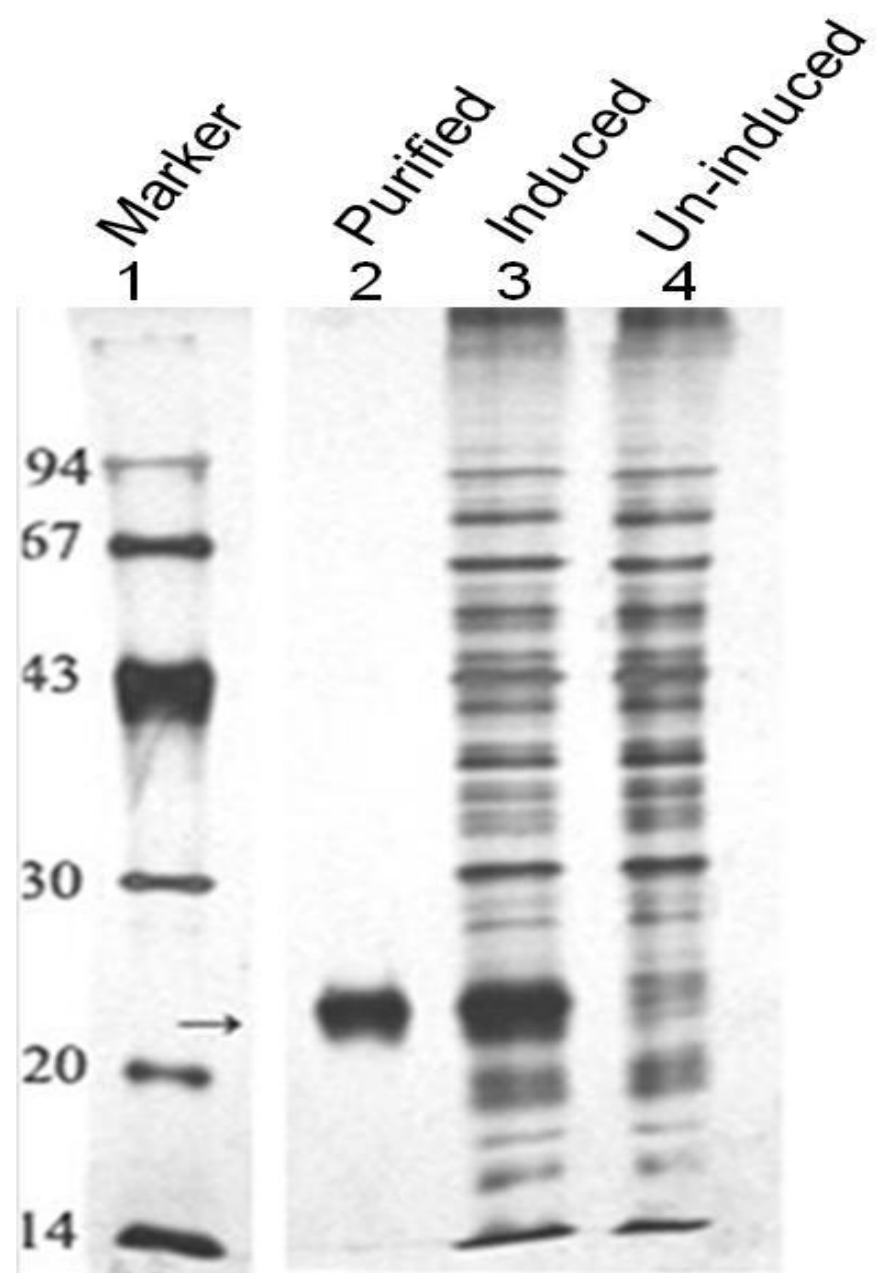

Figure 1. The SDS-PAGE profile of OsDREB1B over-expressed samples: OsDREB1B was amplified by RT-PCR using forwardCATCCATGGAGGTGGAGGAG and reverseTTCAGTGCGAGATTTTGATT primers and cloned in pGEM-T Esay vector. It was further sub-cloned in pET $22 \mathrm{~b}$ at Nco I and Xho I sites followed by transformation in $E$. coli BL21DE3 competent cells. Protein induction was done by addition of 0.5 mM IPTG (isopropyl $\beta$-D-thiogalactoside) for $5 \mathrm{~h}$. Cells were harvested at $6000 \mathrm{rpm}$ for $10 \mathrm{~min}$ at $4^{\circ} \mathrm{C}$. The pellet was washed once with $20 \mathrm{mM}$ TRIS- $\mathrm{HCl}, \mathrm{pH} 8.0$ and finally re-suspended in $500 \mu \mathrm{l}$ of TRIS-HCl, pH 8.0. For checking the expression of protein the re-suspended pellet was boiled in presence of SDSLaemmli buffer and loaded into the gel (lane 3).Then the remaining induced pellet was used for protein purification using nickel chelate affinity matrix as per manufacturer's manual (Qiagen, USA) and finally, the purified protein was checked after running in SDS-PAGE (lane 2). The lane 1 shows the molecular weight of known marker.

of OsDREB1B protein, the CDNA was again sub cloned in pET 22 b vector and over-expressed in Escherichia coil. The over-expressed protein was purified which showed an apparent homogeneity at approximately $23 \mathrm{kDa}$ (Figure 1). Dubouzet et al. (2003) reported that OsDREB1B gene was induced by LT.
A

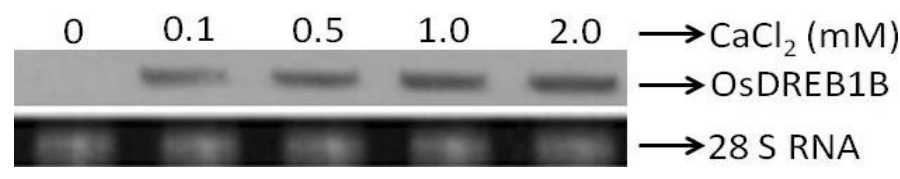

B

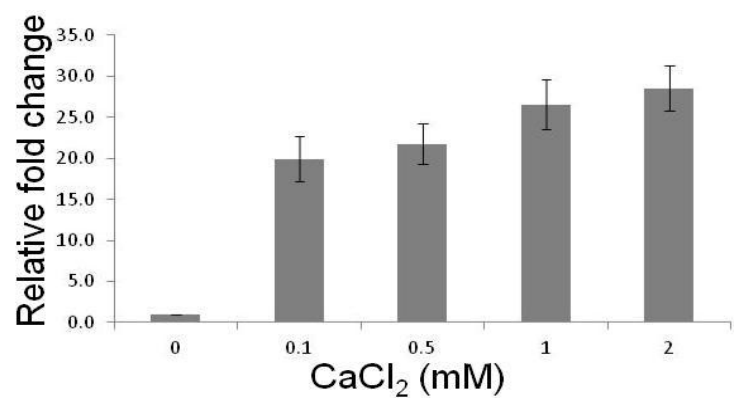

Figure 2. Calcium induces the expression level of OsDREB1B: Seven days old rice seedlings were exposed with various concentrations of $\mathrm{CaCl}_{2}$ for $2 \mathrm{~h}$ at $25^{\circ} \mathrm{C}$. The seedlings were frozen in liquid nitrogen. (A) Northern blot experiment: Total RNA was extracted using the procedure described by Ougham and Davis (1990). $15 \mu \mathrm{g}$ of RNA was resolved on $1.5 \%$ denaturing agarose gel, transferred to $\mathrm{N}^{+}$membrane and was probed with full length CDNA of OsDREB1B. The digits at the top indicate the concentrations of $\mathrm{CaCl}_{2}$ in $\mathrm{mM}$. The expression $28 \mathrm{~S}$ RNA showed the loading control (lower panel). The data presented here was the representation of three different experiments. (B) shows the relative fold change of OsDREB1B mRNA expression data of $(A)$ with respect to control. Data presented here was the mean \pm SD of three independent experiments.

\section{OsDREB1B induction by calcium}

The most upstream event in the LT signaling is the elevated level of cytosolic calcium which follows the activation of ICE and induction of CBF (Chinnusamy et al., 2003). The CBF then activates its downstream targets for resistance to cold. However, a direct co-relation between elevated cytosolic calcium and CBF induction is missing. Here, we studied the expression of an important CBF family gene in response to calcium in absence of LT. Monroy and Dhindsa (1995) reported that the protoplasts of alfalfa need to be incubated for $2 \mathrm{~h}$ at 0.1 $\mathrm{mM} \mathrm{CaCl} 2$ for appreciable amount of calcium infusion. Seven day old Oryza seedlings were exposed to various concentrations $(0.1 \mathrm{mM}$ to $2 \mathrm{mM})$ of $\mathrm{CaCl}_{2}$ for $2 \mathrm{~h}$ at $25^{\circ} \mathrm{C}$. After the end of the treatment, the total RNA was extracted and northern blot experiment was carried out. Figure 2A demonstrates the calcium induced the mRNA expression of OsDREB1B gene in absence of LT. A diagrammatically fold change of mRNA expression was also shown in Figure 2B. Interestingly, it was noted that $0.1 \mathrm{mM}$ calcium significantly induced the OsDREB1B gene. It was also showed that with the increase of calcium concentrations, the induction of OsDREB1B was 


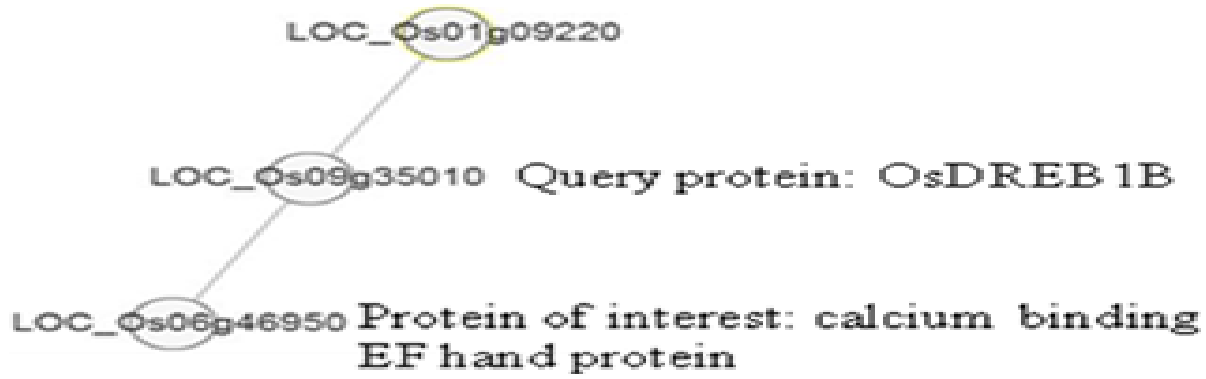

Figure 3. Co-expression analysis of OsDREB1B in abiotic stress: The gene id LOC_Os09g35010 (synonymous to OsDREB1B; AF300972) was used as input data in coexpression analysis at rice array database (http://www.ricearray.org/coexpression/network.shtml; Jung et al., 2008) and was found to be co-expressed with two other proteins in abiotic stress conditions.

further enhanced and maximum induction was observed at $2.0 \mathrm{mM}$ (Figure 2). Thus, the data reveals that calcium induces CBF expression in absence of LT. This also implies that calcium can mimic the effect of $L T$.

\section{Bio-informatics support of the data}

In order to further investigate the inter-relationship of calcium and CBF expression bioinformatics analysis was performed. The co-expression analysis of LOC_Os9g35010.1 (synonymous to OsDREB1B; AF300972) at rice array database (Jung et al., 2008) against abiotic stress network showed two proteins to be co-expressed with OsDREB1B; i) calcium binding EF hand protein (LOC_Os06g46950; marked as protein of interest in the Figure 3); and ii) A putative transposon protein (LOC_Os01g9220). Further, the EF hand calcium binding protein (LOC_Os06g46950) was found to be induced by abiotic stress in microarray database, implying that both the proteins (OsDERB1B and EF hand calcium binding protein) are co-expressed by abiotic stress (Jung et al., 2008). EF hand is a helix-loop-helix structural domain present in the calcium binding proteins. In plants calcium sensors can be broadly divided into four major classes: A) calmodulin (CAM); B) CAM like and other EF hand containing calcium binding protein; C) calcium regulated protein kinases; and D) calcium binding protein without EF hand (Reddy, 2000; White, 2000; Knight, 2000). Here, we predict that increased calcium might be sensed by EF hand calcium binding protein, which might help in CBF induction. It has earlier been reported that a calcium interacting protein CAMTA (Calmodulin binding Transcriptional Activators) regulates CBF expression by binding to CBF2 promoter in $A$. thaliana (Doherty et al., 2009).

\section{Conclusion}

In this report, we cloned and over-expressed a divalent metal ion (calcium) induced CBF gene (OsDREB1B). The OsDREB1B gene was up-regulated by calcium in absence of LT, appearing that calcium could mimic the effect of LT. The result clearly demonstrates a link between calcium and CBF induction. The data also suggest that low amount of calcium $(0.1 \mathrm{mM})$ is enough to induce the OsDREB1B gene, which implies that calcium enriched environment might be able to prevent potential damages to rice from LT shock. However, this needs to be further investigated. The bioinformatics analysis suggests that the induction of OsDREB1B by calcium might be mediated through an EF hand calcium binding protein.

\section{ACKNOWLEDGEMENT}

We are grateful to KIIT University, Bhubaneswar, Odisha, India for financial support for the work.

\section{REFERENCES}

Chinnusamy V, Ohta M, Kanrar S, Lee B, Hong X, Agarwal M, Zhu JK (2003). ICE1: a regulator of cold-induced transcriptome and freezing tolerance in Arabidopsis. Genes Dev. 17:1043-1054.

Doherty CJ, Buskirk HA, Myers SJ, Thomashow MF (2009). Roles for Arabidopsis CAMTA Transcription Factors in Cold-Regulated Gene Expression and Freezing Tolerance. Plant Cell 21:972-984.

Dubouzet JG, Sakuma Y, Ito Y, Kasuga M, Dubouzet EG, Miura S, Seki M, Yamaguchi-Shinozaki K, Shinozaki K (2003). OsDREB genes in rice, Oryza sativa L., encode transcriptional activator that function in drought, high salt and cold-responsive gene expression, Plant J. 33:751-763.

Gilmour SJ, Zarka DG, Stockinger EJ, Salazar MP, Houghton JM, Thomashow MF (1998). Low temperature regulation of Arabidopsis CBF family of AP2 domain transcriptional activators as an early step in cold-induced COR gene expression. Plant J. 16:433-442.

Jung KH, Dardick C, Bartley LE, Cao P, Phetsom J, Canlas P, Seo YS, Shultz M, Ouyang S, Yuan Q, Frank BC, Ly E, Zheng L, Jia Y, Hsia AP, An K, Chou HH, Rocke D, Lee GC, Schnable PS, An G, Buell CR and Ronald PC (2008). Refinement of light-responsive transcript lists using rice oligonucleotide arrays: evaluation of generedundancy. PLoS ONE, 3:e3337.

Knight $\mathrm{H}$ (2000). Calcium signaling during abiotic stress in plants. Int. Rev. Cytol. 195:269-324. 
Monroy AF, Dhindsa RS (1995). Low temperature signal transduction: Induction of cold acclimation-specific genes of alfalfa by calcium at $25^{\circ} \mathrm{C}$. Plant Cell 7:321-331.

Ougham HJ, Davies TGE (1990). Leaf development in Lolium temulentum: gradients of RNA compliment and plastid and nonplastid transcript. Physiol. Plant 79:331-338.

Reddy ASN (2000). Calcium: silver bullet in signaling. Plant Sci. 160:381-404.

Thomashow MF (2010). Molecular Basis of Plant Cold Acclimation: Insights Gained from Studying the CBF Cold Response Pathway. Plant Physiol. 154:571-577.
Vogel JT, Zarka DG, Buskirk HA, Fowler SG, Thomashow MF (2005). Roles of the CBF2 and ZAT12 transcription factors in configuring the low temperature transcriptome of Arabidopsis. Plant J. 41:195-211.

White PJ (2000). Calcium channels in higher plants. Biochim. Biophys. Acta. 1465:171-189.

Zhao H, Liu W, Hua Y, Liu X, He Y (2008). Cis-regulatory elementbased genome-wide identification of DREB1/CBF targets in Arabidopsis. Prog. Nat. Sci. 18:579-583. 